\title{
Assessing prognostic value of early tumor shrinkage and depth of response in first-line therapy for patients with advanced unresectable pancreatic cancer
}

\author{
Xiaojuan Yang ${ }^{1}$, Xinghong Xian ${ }^{1}$, Yongsheng Wang ${ }^{1}$ and Meng Qiu ${ }^{2 *}$
}

\begin{abstract}
Background: The prognostic potential of early tumor shrinkage (ETS) and depth of response (DpR) in pancreatic cancer (PC) is unclear. Here, we recruited 90 patients with recurrent and metastatic PC (RMPC) who had received chemotherapy as first-line therapy to assess the prognostic potential of these markers.

Methods: ETS is characterized as a $\geq 20 \%$ depletion in the sum-of-the-longest-diameters (SLD) of measurable tumor lesions at 6-12 weeks than the baseline. DpR is the maximum shrinkage (\%) from the baseline to nadir. We evaluated corrections in ETS and DpR with survival.

Results: Of the 63 patients in which ETS assessment was possible, 21 (33.3\%) achieved ETS. We found a significant association between the incidence of ETS and an improved rate of progression-free survival (PFS; 6.5 vs. 2.2 months; $p<0.001)$ and overall survival (OS; 12.1 vs. 6.0 months; $p=0.014)$. The median value of DpR was $-23.66 \%$. DpR was also related to improved PFS (9.3 vs. 3.1 months; $p<0.001)$ and OS (18.2 vs. 7.3 months; $p<0.001)$. Patients who had distant metastasis, not local recurrence, with ETS showed markedly better outcomes. In a multivariate model, both ETS and DpR were independent predictors of OS in the whole population.

Conclusions: ETS and DpR may predict favorable outcomes for RMPC patients who had received chemotherapy as first-line therapy, independent of the agents used. Further studies on the exploratory analyses of the optimum ETS cut-off value in recurrent PC patients to predict favorable clinical outcomes are required.
\end{abstract}

Keywords: ETS, DpR, Recurrence, Metastasis, Pancreatic cancer, Prognosis

\section{Background}

The 5-year survival rate of pancreatic cancer (PC), a fatal disease, is $9 \%$ for all stages combined, and it ranks seventh in terms of global mortality due to cancer [1, 2]. Despite early diagnosis, it has a post-surgery 5 -year survival rate $<20 \%$ and approximately $80 \%$ of the cases

\footnotetext{
*Correspondence: qiumeng33@hotmail.com

${ }^{2}$ Division of Abdominal Oncology, Cancer Center, West China Hospital,

Sichuan University, No. 37 Guo Xue Xiang, Chengdu 610041, China

Full list of author information is available at the end of the article
}

are known to relapse within 2 years [3-5]. Additionally, patients with metastasized PC have a 5-year survival rate of $3 \%$ [5].

Recurrent and metastatic pancreatic cancer (RMPC) remains an incurable disease. Currently, the systemic treatment options involve gemcitabine monotherapy or combined chemotherapy, including gemcitabine-based regimens and fluorouracil (5FU)-based regimens as the first-line therapy $[6,7]$. Despite the availability of various types of treatment regimens for advanced PC, there is a lack of factors that define the precocity or depth of 


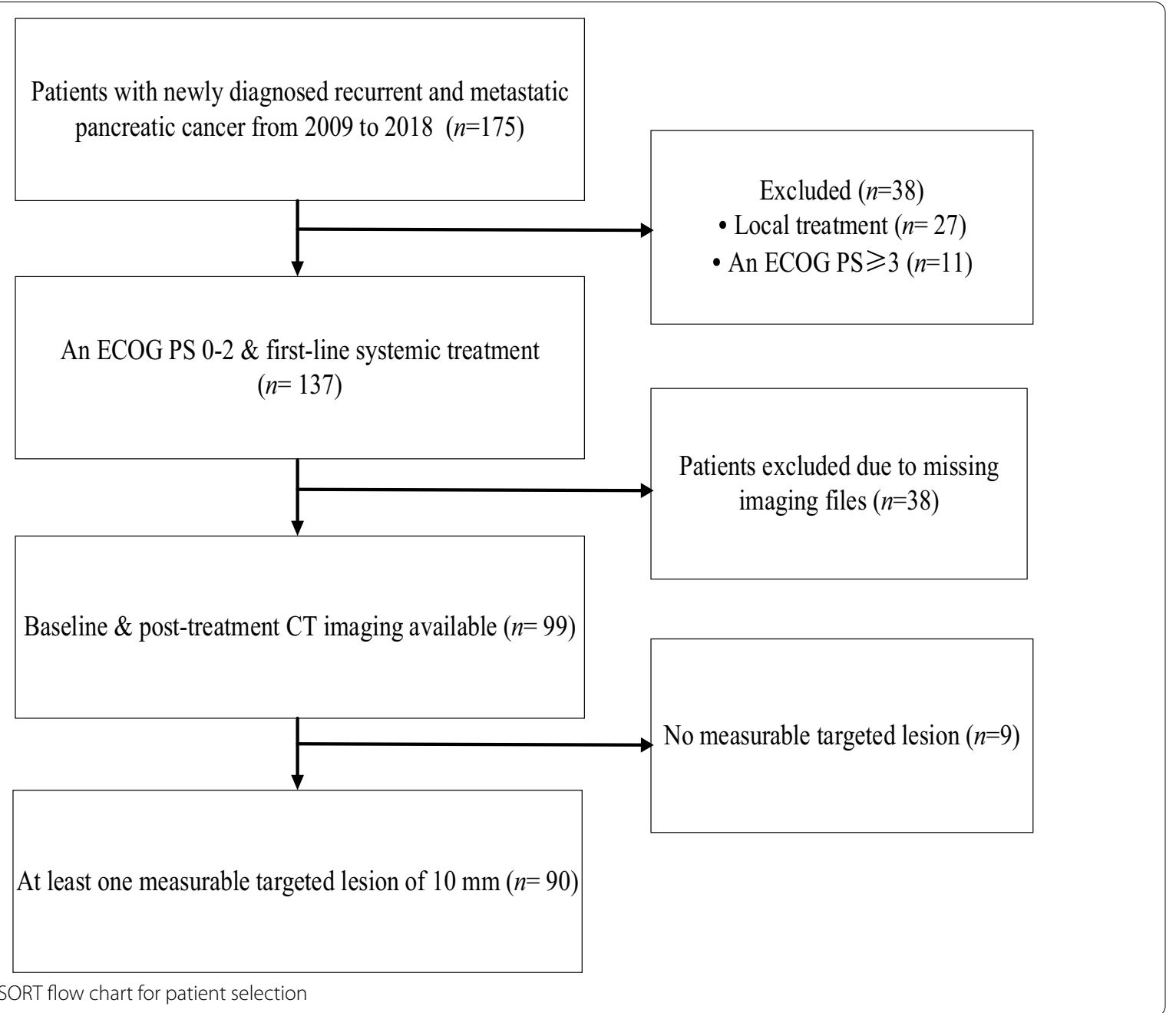

tumor regression and can reliably anticipate prognosis as the first-line of therapy.

Early tumor shrinkage (ETS) implies a $\geq 20 \%$ depletion in tumor burden measured after treatment initiation compared with that evaluated at the baseline [8]. Depth of response (DpR) is classified as the largest decrease (\%) in the tumor size, calculated based on the reconstructed volume or longest diameters at the nadir than the baseline [9]. The post-hoc analysis of three randomized trials (OPUS, CRYSTAL, and FIRE-1) showed that ETS within 7-8 weeks post-treatment initiation was significantly related to a longer overall survival (OS) and progression-free survival (PFS) $[9,10]$. Additionally, the post-hoc analyses of TRIBE, PEAK, and FIRE-3 trials demonstrated that DpR $>$ median value was linked to a longer PFS, post-progression survival (PPS), and OS [11-13]. Thus, these studies confirmed that both ETS and DpR were related to a good prognosis in cases of metastatic colorectal cancer
(mCRC), irrespective of the first-line systematic therapy received.

However, there is limited information available on the function of DpR and ETS and in the outcome prediction of RMPC. The first investigational study that examined ETS as a potentially favorable outcome predictor involved 59 subjects with advanced PC who were being treated with FOLFIRINOX (5-fluorouracil, irinotecan, and oxaliplatin) [14]. It reported that patients who achieved an ETS had a median value of OS and PFS as 24.0 months and 9.0 months, respectively, compared with 9.1 and 4.2 months for the non-ETS patients. The ETS was statistically significantly with PFS $(p=0.020)$ in multivariate analysis but was not statistical significance with OS $(p=0.065)$ [14]. Recently, a retrospective study conducted by Caterina V et al. on 138 patients with metastatic PC receiving either FOLFOXIRI (5-fluorouracil, irinotecan, and oxaliplatin) or Gemcitabine plus Nabpaclitaxel. The results showed that DpR and ETS were 
Table 1 Baseline demographic and clinical characteristics

\begin{tabular}{ll}
\hline Characteristics & $\begin{array}{l}\text { Patients } \\
n=90\end{array}$ \\
\cline { 2 - 2 } & $n$
\end{tabular}

\section{Age, years}

Median

Range

$$
<65
$$$$
\geq 65
$$$$
\text { Sex }
$$$$
\text { Male }
$$

Female

ECOG performance status

0

1

2

Histology

Adenocarcinoma

Adenosquamous carcinoma

Tumor site

Head-uncinate process

Body-tail

Multifocal

Synchronous disease

$$
\text { Yes }
$$

No

No. of metastatic sites

$$
1-2
$$$$
\text { 3-4 }
$$

Localization of metastasis

$$
\text { Liver }
$$

Peritoneal

$$
\text { Lung }
$$

Bones

Local recurrence (including regional lymph node metastases)

Previous treatments

Radical surgery

Adjuvant chemotherapy

Ca19.9 (KU/L)

$$
\leq \text { ULN }
$$

$>$ ULN

Biliary stent

Yes

No

$\mathrm{HDL}-\mathrm{C}(\mathrm{mmol} / \mathrm{L})$

$$
>0.9
$$

$$
\leq 0.9
$$

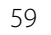

63

27

57

33

\section{4}

27

19

89

1

41

47

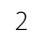

47

26

61

12

61

13

6

2

17

Ca19.9 carbohydrate antigen 19-9, DpR depth of response, ECOG Eastern Cooperative Oncology Group, ETS early tumour shrinkage, IPMN intraductal papillary mucinous neoplasm, ULN upper limit of normal, HDL-C High-density lipoprotein cholesterol
Table 2 Distribution of ETS and DpR

\begin{tabular}{lll}
\hline ETS and DpR cut-offs & Patients $(\boldsymbol{n})$ & $\%$ \\
\hline ETS & 63 & - \\
$\geq 20 \%$ & 21 & 33.3 \\
$<20 \%$ & 42 & 66.7 \\
DpR & 62 & - \\
I quartile (- 100\% to - 37.98\%) & 16 & 25.4 \\
II quartile (- 37.98\% to - 23.66\%) & 15 & 23.8 \\
III quartile (- 23.66\% to + 15.28\%) & 15 & 23.8 \\
IV quartile (+ 15.28\% to + $67.89 \%)$ & 16 & 25.4 \\
$<$ Median & 31 & 50 \\
$\geq$ Median & 31 & 50 \\
\hline DpR depth of response ETS &
\end{tabular}

48.9

30.0

21.1

98.9

1.1

45.6

52.2

2.2

64.4

35.6

83.6

16.4

67.8

14.4

6.7

2.2

18.9

47.8

26.7

16.7

83.3

3.3

96.7

85.6

14.4 strongly related to OS and PFS in PC patients treated with FOLFOXIRI; however, no statistical correlation was observed in the Gemcitabine plus Nab-paclitaxel cohort [15]. Therefore, previous studies reported a conflict between the association of ETS and OS, indicating the need for further studies. Additionally, the prognostic potential of DpR and ETS in advanced PC patients who received chemotherapy as the first-line therapy is not completely understood, and thus, it is unclear whether the prognostic potential is independent of treatment administration.

Here, we examined the prognostic potential of ETS and $\mathrm{DpR}$ in RMPC patients who received chemotherapy as the first-line therapy, irrespective of their treatment regimens.

\section{Methods \\ Patients}

We reviewed RMPC patients at the West China Hospital of Sichuan University database between 2009 and 2018 (Fig. 1). The enrolled patients were $\geq 18$ years, had an Eastern Cooperative Oncology Group (ECOG) performance status (PS) of 0,1 , or 2 , and had histopathologically confirmed recurrent and unresectable or metastatic PC. According to the Response Evaluation Criteria in Solid Tumors (RECIST), version 1.1 [16], eligible patients should have at least one $10 \mathrm{~mm}$ measurable targeted lesion, had at least one first-line systemic treatment at our institution, and had done their tumor assessments by Computed Tomography (CT) at least once. Cases without available imaging within 28 days before treatment initiation and lacking complete clinicopathologic and follow-up data were excluded. Patients who had a history of another major cancer, endocrine pancreatic carcinoma, and were pregnant or breastfeeding were also excluded. 


\section{Evaluation of ETS and DpR}

The investigators used the RECIST version 1.1 to assess the tumor responses [16]. The sum-of-the-longest-diameters (SLD) of RECIST tumor lesions at baseline and after treatment initiation were analyzed. ETS was classified as $\mathrm{a} \geq 20 \%$ depletion in the SLD at 6-12 weeks after treatment initiation [17]; non-ETS included a minor shrinkage (a decrease by 0 to $19 \%$ ), tumor growth, and new metastatic lesions [8]. DpR was characterized as the maximal decrease (\%) from the baseline to nadir, without the appearance of novel lesions or the growth of non-target lesions $[12] . \mathrm{DpR}=[(\mathrm{SLD}$ at nadir $)-($ SLD at baseline $)]$ $/$ (SLD at baseline $) \times 100 \%$ [15]. We evaluated ETS as both a continuous and a binary $(\geq 20 \%$ vs. $<20 \%)$ variable. Also, DpR was treated either as continuous, or as an ordinal (with four levels based on quintile distribution), or as a binary ( $\geq$ median vs. $<$ median) variable. The $D p R$ quartiles were classified as follows: quartile I: $-100 \%$ to $-37.98 \%$; quartile II: $-37.98 \%$ to $-23.66 \%$; quartile III: $-23.66 \%$ to $+15.28 \%$; quartile IV: $+15.28 \%$ to $+67.89 \%$ (“+”, i.e. "increase of tumor," “-, i.e. "tumor shrinkage”) $[12,15]$.

\section{Study endpoints}

The co-primary endpoints included the relationship between ETS/DpR and PFS/OS among all the participants. We defined median OS as the duration between treatment initiation (day 1) to the first documented death, whatever the cause and median PFS was defined as time from the first day of treatment to the date of the first documented tumor growth or death, whatever the cause. We censored patients who were alive and who had no disease progression at their last follow-up visit or at the date of their last radiologic assessment. Additionally, those patients were also censored whose survival time, including PFS and OS, were unavailable.

\section{Statistical analysis}

We used Kaplan-Meier curves to estimate the link between ETS/DpR with survival, including PFS/OS, and compared it with the log-rank test (two-sided). For OS, each factor was initially assessed for their prognostic effect using univariate Cox proportional hazards regression analysis (enter method) [17]. Variables with $p<0.2$ in the univariate analyses were selected as explanatory determinants for a stepwise multivariate Cox proportional hazards regression model. Nominal variables were presented as percentages. The Cox regression model was used to calculate the hazard ratios (HRs) and 95\% confidence intervals (CIs). A $p$-value $<0.05$ for a two-sided test was regarded as statistically significant. All statistical analyses were done using IBM SPSS v25.0 (SPSS Inc., USA) and GraphPad Prism v8.0.2 (GraphPad Software, Inc., USA.).

Table 3 Association between clinicopathological features and survival parameters

\begin{tabular}{|c|c|c|c|c|}
\hline \multirow[t]{2}{*}{ Clinicopathological features } & \multicolumn{2}{|l|}{ PFS } & \multicolumn{2}{|l|}{ os } \\
\hline & HR $(95 \% \mathrm{Cl})$ & $P$-value & $\mathrm{HR}(95 \% \mathrm{Cl})$ & $P$-value \\
\hline Age ( $\geq 65$ years) & $0.741(0.460-1.192)$ & 0.216 & $0.653(0.377-1.131)$ & 0.128 \\
\hline ECOG PS (2) & $9.725(4.78-19.785)$ & $<0.001$ & $5.200(2.711-9.974)$ & $<0.001$ \\
\hline Gender (female) & $1.037(0.665-1.618)$ & 0.872 & $1.192(0.708-2.006)$ & 0.508 \\
\hline Sites of metastases (liver) & $1.425(0.909-2.235)$ & 0.123 & $1.948(1.088-3.487)$ & 0.025 \\
\hline Sites of metastases (lung) & $1.105(0.478-2.555)$ & 0.815 & $0.557(0.169-1.839)$ & 0.337 \\
\hline Sites of metastases (peritoneum) & $1.646(0.900-3.011)$ & 0.106 & $1.002(0.508-1.976)$ & 0.995 \\
\hline $\begin{array}{l}\text { Local recurrence (including } \\
\text { regional lymph node metastases) }\end{array}$ & $1.237(0.636-2.407)$ & 0.531 & $1.140(0.486-2.673)$ & 0.763 \\
\hline Number of metastatic sites $(\geq 3)$ & $2.239(1.197-4.186)$ & 0.012 & $2.090(1.027-4.252)$ & 0.042 \\
\hline Synchronous disease (no) & $0.860(0.533-1.390)$ & 0.539 & $0.815(0.460-1.445)$ & 0.484 \\
\hline Tumor site (body-tail) & $1.057(0.741-1.508)$ & 0.759 & $0.797(0.533-1.192)$ & 0.270 \\
\hline Surgery for primary tumor (yes) & $0.782(0.513-1.194)$ & 0.255 & $0.815(0.492-1.349)$ & 0.425 \\
\hline CA-199 (KU/L) (> ULN) & $1.532(0.887-2.645)$ & 0.126 & $1.415(0.737-2.716)$ & 0.297 \\
\hline $\mathrm{HDL}-\mathrm{C}(\mathrm{mmol} / \mathrm{L})(>0.9)$ & $1.560(0.819-2.971)$ & 0.177 & $1.104(0.570-2.138)$ & 0.770 \\
\hline ETS $(\geq 20 \%)$ & $0.523(0.315-0.868)$ & $<0.001$ & $0.699(0.387-1.263)$ & 0.014 \\
\hline ETS (as a continuous variable) & $1.015(1.010-1.020)$ & $<0.001$ & $1.011(1.006-1.017)$ & $<0.001$ \\
\hline $\mathrm{DpR}$ ( $\geq$ median) & $0.163(0.092-0.290)$ & $<0.001$ & $0.252(0.132-0.480)$ & $<0.001$ \\
\hline DpR (as a continuous variable) & $1.035(1.025-1.046)$ & $<0.001$ & $1.022(1.013-1.031)$ & $<0.001$ \\
\hline
\end{tabular}

Bold values indicate statistical significance at $P<0.05$

Ca19.9 carbohydrate antigen 19-9, Cl confidence interval, DpR depth of response, ECOG Eastern Cooperative Oncology Group, ETS early tumour shrinkage, ULN upper limit of normal, HDL-C High-density lipoprotein cholesterol 

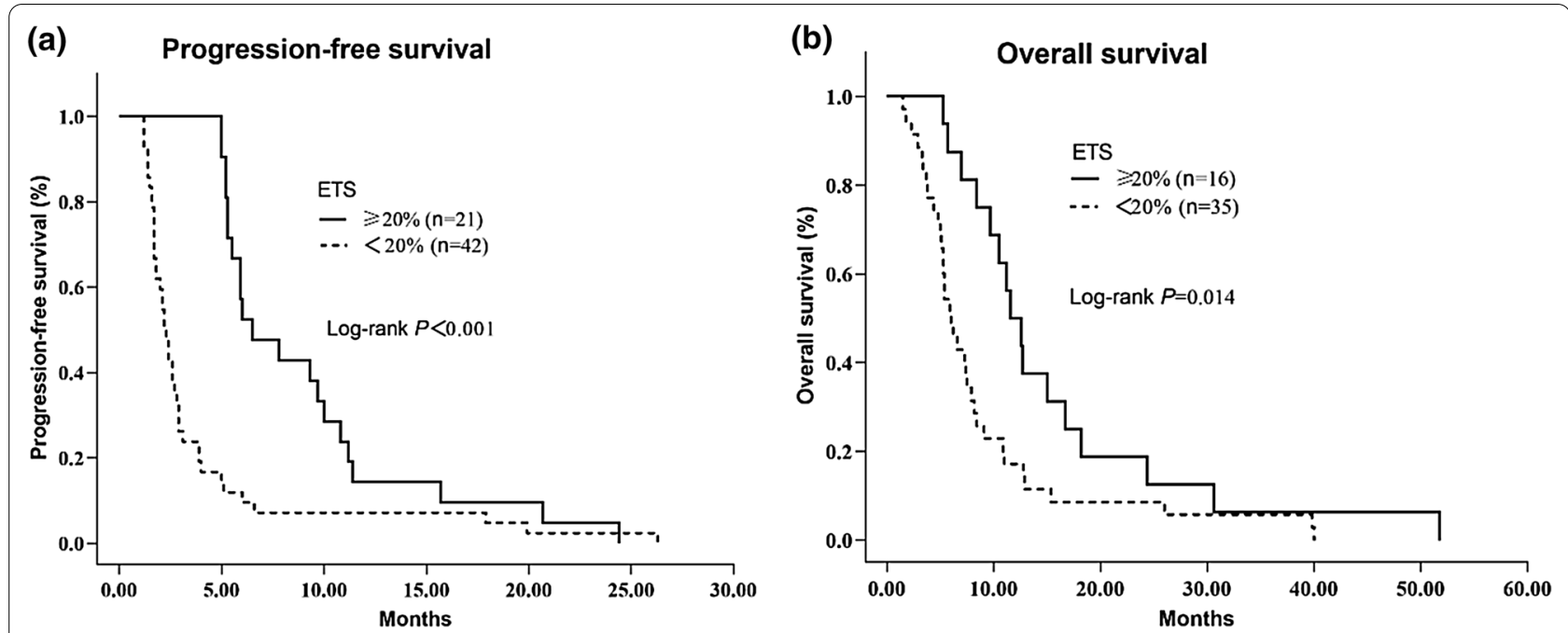

Fig. 2 a Kaplan-Meier survival curves for PFS in ETS and non-ETS patients in the entire population b Kaplan-Meier curves for OS in ETS and non-ETS patients in the entire population

\section{Results}

\section{Patient characteristics at baseline}

One hundred seventy-five patients were diagnosed with RMPC between 2009 and 2018, of which 90 were included based on the study criteria (Fig. 1). Table 1 lists the baseline characteristics of the study population.

\section{Treatment regimens and efficacy}

The present analysis included 90 patients with different first-line treatment regimens. Specifically, 41 patients (46\%) were treated with gemcitabine plus S-1 (GS); 14 patients (16\%) received FOLFIRINOX. Approximately $8(9 \%)$ and $6(7 \%)$ patients were administered GEMOX (Gemcitabine and Oxaliplatin) and Gemcitabine plus Nab-paclitaxel, respectively. Additionally, there were 11 less frequently chosen regimens (data are given in Additional file 1: Table S1). Furthermore, one patient was given three cycles of GS and three cycles of Gemcitabine plus Nab-paclitaxel.

Each patient received the assigned therapy for four cycles in the median (1-10), with a median follow-up of 12.2 months. Amongst the study participants, the median OS and PFS were 8.8 and 4.4 months, respectively. The disease control rate (DCR) was 52.2\% (47 patients), and the objective response rate (ORR) was 28.9\% (26 patients, 22 achieving partial responses and 4 complete responses), and the based on RECIST v1.1 criterion. The death of 66 patients $(73.3 \%)$ was recorded during the analysis. Meanwhile, the disease progressed in 89 patients (98.9\%), of which $44(49.4 \%)$ received secondline therapy. There were 18 different types of regimens for second-line therapy, of which the most frequently chosen was FOLFIRINOX (31.8\%). Three patients (3.3\%) had a biliary stent who had a poor prognosis with a median OS and PFS of 6.6 months and 1.4 months, respectively.

\section{ETS and DpR}

Of the 90 patients, 63 were evaluated for the ETS. The development of new metastatic lesions was observed in 17 patients at 6-12 weeks after treatment initiation. Among the remaining 46 patients, the median reduction of the SLD from baseline was $-10.22 \%$, and 21 patients (33.3\%) achieved ETS.

Among 62 assessable patients, the median DpR was $-23.66 \%$ (from -100 to +67.89 ). Table 2 reports the quartile distribution of DpR, considering DpR as a continuous variable.

\section{Prognostic factors univariate analyses}

Amongst the unselected patients, both ETS (achieving ETS and ETS as a continuous variable) and DpR ( $\mathrm{DpR} \geq$ median and $\mathrm{DpR}$ as as a continuous variable) had greater univariate analyses values, which were significantly related to better PFS and OS (all $p<0.05$ ) (Table 3 ).

For the univariate analyses, variables that were significantly correlated to unfavorable PFS included ECOG PS 2 (median PFS 1.8 vs. 5.3 months, $p<0.001$ ) and the total metastatic sites $\geq 3$ (median PFS 2.3 vs. 5.0 months, $p=0.012$ ), whereas ECOG PS 2 (median OS 4.5 vs. 11.1 months, $p<0.001$ ), total metastatic sites $\geq 3$ (median OS 7.7 vs. 9.5 months, $p=0.042$ ), and liver metastasis (median OS 4.0 vs. 10.9 months, $p=0.025$ ) were considerably related to inferior OS (Table 3 ). 

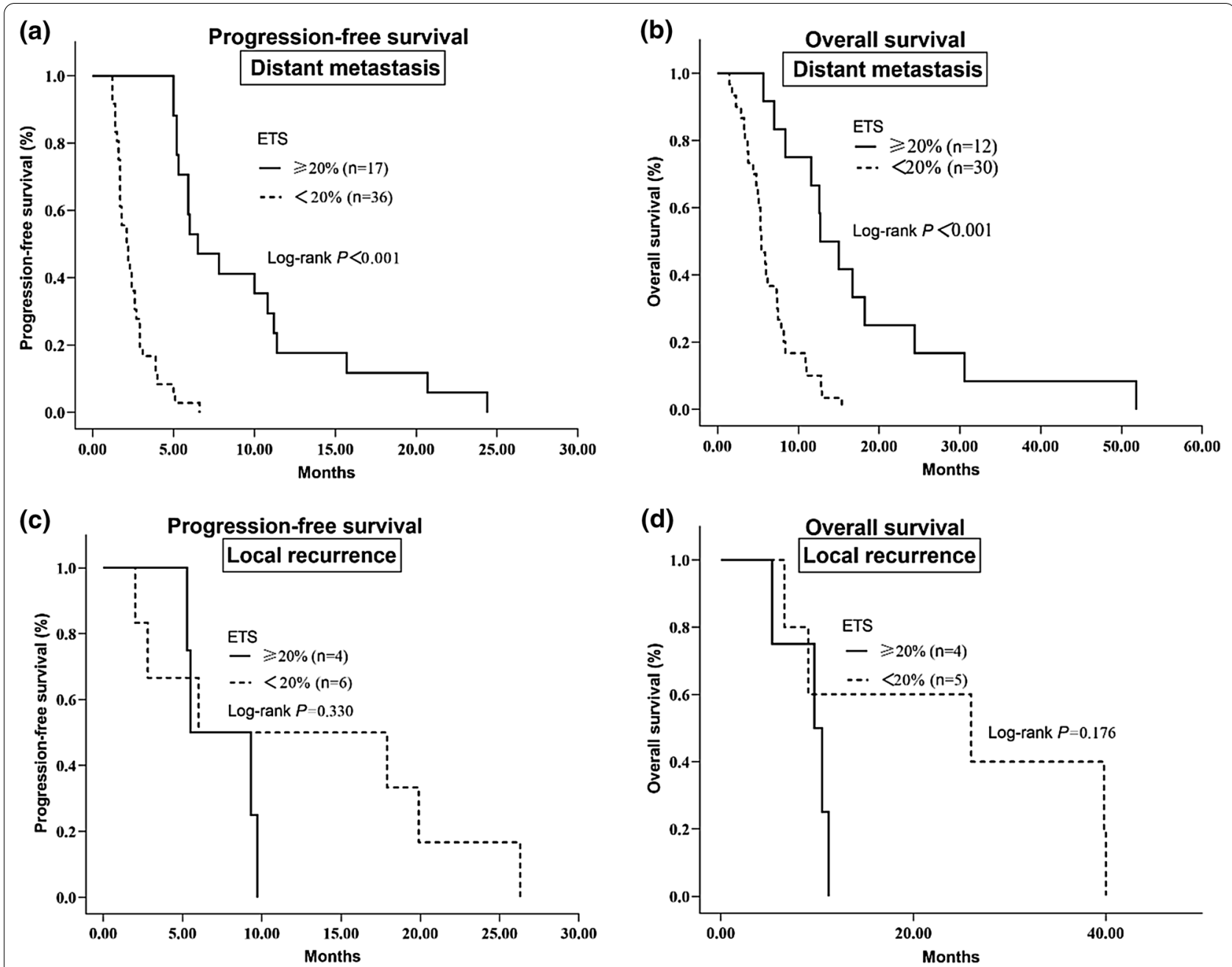

Fig. 3 a Kaplan-Meier survival curves for PFS in ETS and non-ETS patients in patients with distant metastasis $\mathbf{b}$ Kaplan-Meier curves for OS in ETS and non-ETS patients in patients with distant metastasis c Kaplan-Meier curves for PFS in ETS and non-ETS patients in patients with local recurrence d Kaplan-Meier curves for OS in ETS and non-ETS patients in patients with local recurrence

ETS and its stratification analysis: correlation with survival Amongst all the patients, patients achieving ETS $(n=21)$ exhibited an improved PFS ( $n=63 ; 6.5$ vs 2.2 months, HR $0.523, p<0.001)$ and OS (12.1 vs 6.0 months, HR 0.699, $p=0.014$ ) compared with the non- ETS patients (Table 3, Fig. 2).

Among the patients evaluated for ETS, 53 patients had distant metastasis, of which 17 (32.1\%) achieved an ETS; while 10 patients had local recurrence, of which 4 reached an ETS. For the stratification analysis, among patients with distant metastasis, evaluation of PFS (6.5 vs. 2.1 months, HR $0.248, p<0.001)$ and OS (13.9 vs. 5.4 months, HR $0.315, p<0.001$ ) favored ETS over nonETS patients (Fig. 3). However, patients with local recurrence showed no significant association between ETS with both PFS ( $p=0.330)$ and OS $(p=0.176)$ (Fig. 3$)$.

\section{DpR and its stratification analysis related to survival}

Amongst the study participants who were assessed, DpR, both as a discrete and a continuous variable, showed a strong link to both OS and PFS (Table 3, Fig. 4).

Among the patients evaluated for DpR, 48 had distant metastasis, while the remaining 14 had local recurrence. For the stratification analysis, both in patients with local recurrence and distant metastasis, a deeper response was significantly linked to PFS and OS ( $p<0.05)$ (Fig. 5).

\section{Prognostic factors multivariate analyses}

The multivariable analysis performed using the Cox proportional hazards regression Model revealed stronger associations between both $\mathrm{DpR}$ as a continuous variable $(p<0.001)$ with a better PFS and ECOG PS 2 $(p=0.041)$ with a shorter PFS after adjusting for potential 

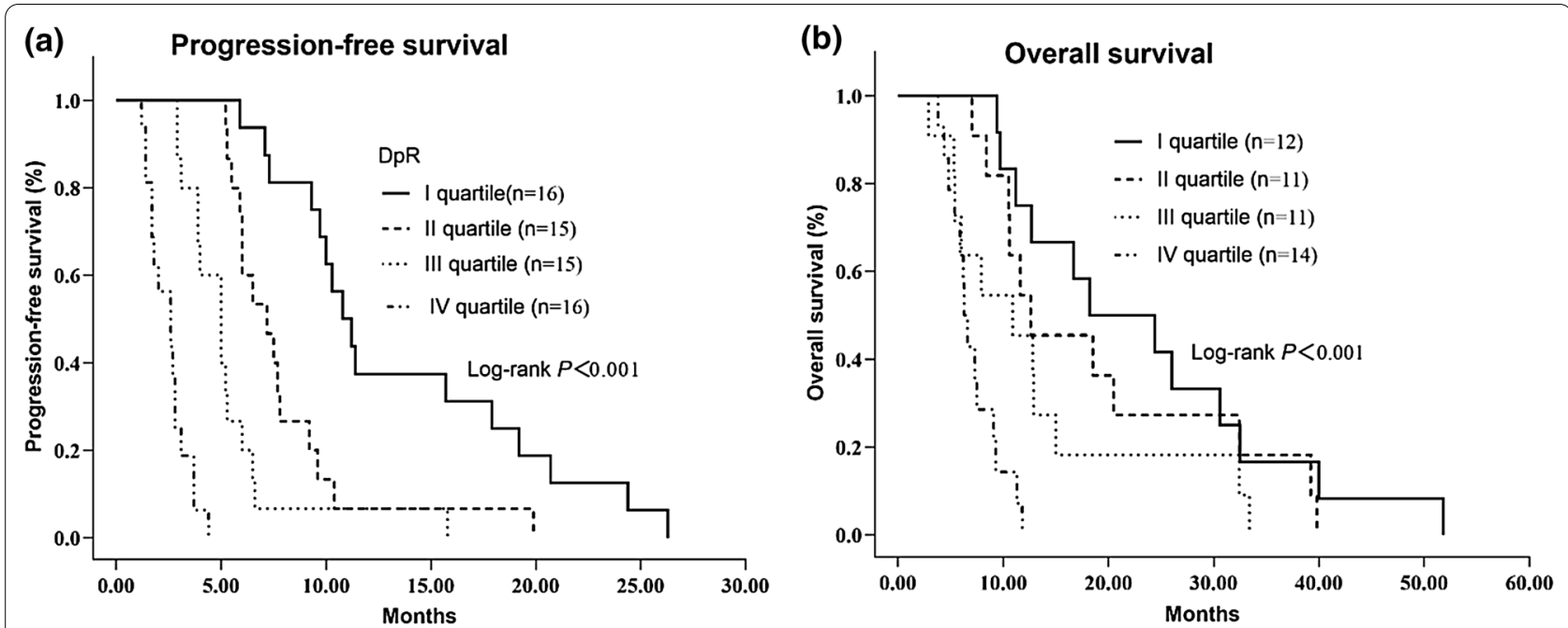

Fig. 4 a Kaplan-Meier survival curves for PFS according to DpR quartiles in the entire population b Kaplan-Meier curves for OS according to DpR quartiles in the entire population

confounding variables. For OS, DpR $(p<0.001)$ and ETS $(p=0.001)$ both were determined to be independent prognostic factors. Furthermore, total number of metastatic sites $\geq 3$ was associated with a poor prognosis for OS $(p=0.003)$ (Table 4$)$.

\section{Discussion}

This study is the first report to examine the prognostic potential of ETS and DpR in first-line therapy for patients with RMPC, irrespective of the treatment regimens. The results of this analysis demonstrated that both ETS and $\mathrm{DpR}$ were significant independent predictors of a longer OS and were not dependent on the first-line treatment received. Moreover, while we observed a strong relationship between ETS and PFS/OS in patients with distant metastasis, a similar level of significance was not reached in patients with local recurrence; thus, suggesting that achieving an ETS $>20 \%$ might not be a long-term outcome predictor for patients with local recurrence.

In this study, we set the cut-off value at $20 \%$ for the optimal distinction between ETS and non-ETS, based on previous reports with advanced PC $[14,15]$. The present study showed that $33.3 \%$ of the patients experienced ETS, which was consistent with the results of Kaga et al. (25.5\%) [14] and Vivaldi et al. (35.5\%) [15]. The median DpR was $-23.66 \%$, which was also consistent with the previously reported percentages in the first published report to establish the prognostic value of $\mathrm{DpR}$ in PC[15].

However, previous two studies reported some conflicts $[14,15]$. Kaga et al. reported that the ETS was not a independent predictor of OS [14]. Caterina et al. showed that $\mathrm{DpR}$ and ETS were significant associations with OS and
PFS in one cohort; however, no statistical correlation was observed in the other cohort [15]. Our study indicated that both ETS and DpR were significant independent predictors of a longer OS. On conducting a multivariate regression analysis, including the metastatic sites, one of the available surrogate measures of tumor burden [10], DpR constituted an independent positive predictive factor of PFS and OS, but ETS was only maintained in our model involving OS. This suggested that the impact of ETS (versus non-ETS) was considerably higher on OS compared with PFS, consistent with the results for metastatic colorectal cancer from a review conducted by Heinemann et al. [18]. In the univariate analyses, ETS was not only associated with OS but also with PFS. One possible reason was that apart from a more favorable prognosis, ETS also distinguished patients who were highly sensitive to treatment from a heterogeneous group and identified patients who were early responders, which was also true for metastatic colorectal cancer $[8,18-21]$. Therefore, in clinical trials, in order to prompt drug development and potentially orientate treatment decisions, such an endpoint would be extremely appealing. In routine practice, ETS $\geq 20 \%$ as a simple, reproducible parameter may predict outcomes and show the advantage of earlier assessment compared to RECIST response. Additionally, recent reports on mCRC claimed that presence of rapid and deep tumor shrinkage were linked with clear benefits in terms of rapidly relieving tumourrelated symptoms, improving quality of life (QOL), delaying tumor progression and predictors of proceeding to conversion surgery $[12,22]$. However, it is unclear whether these potential values exist in pancreatic cancer, 

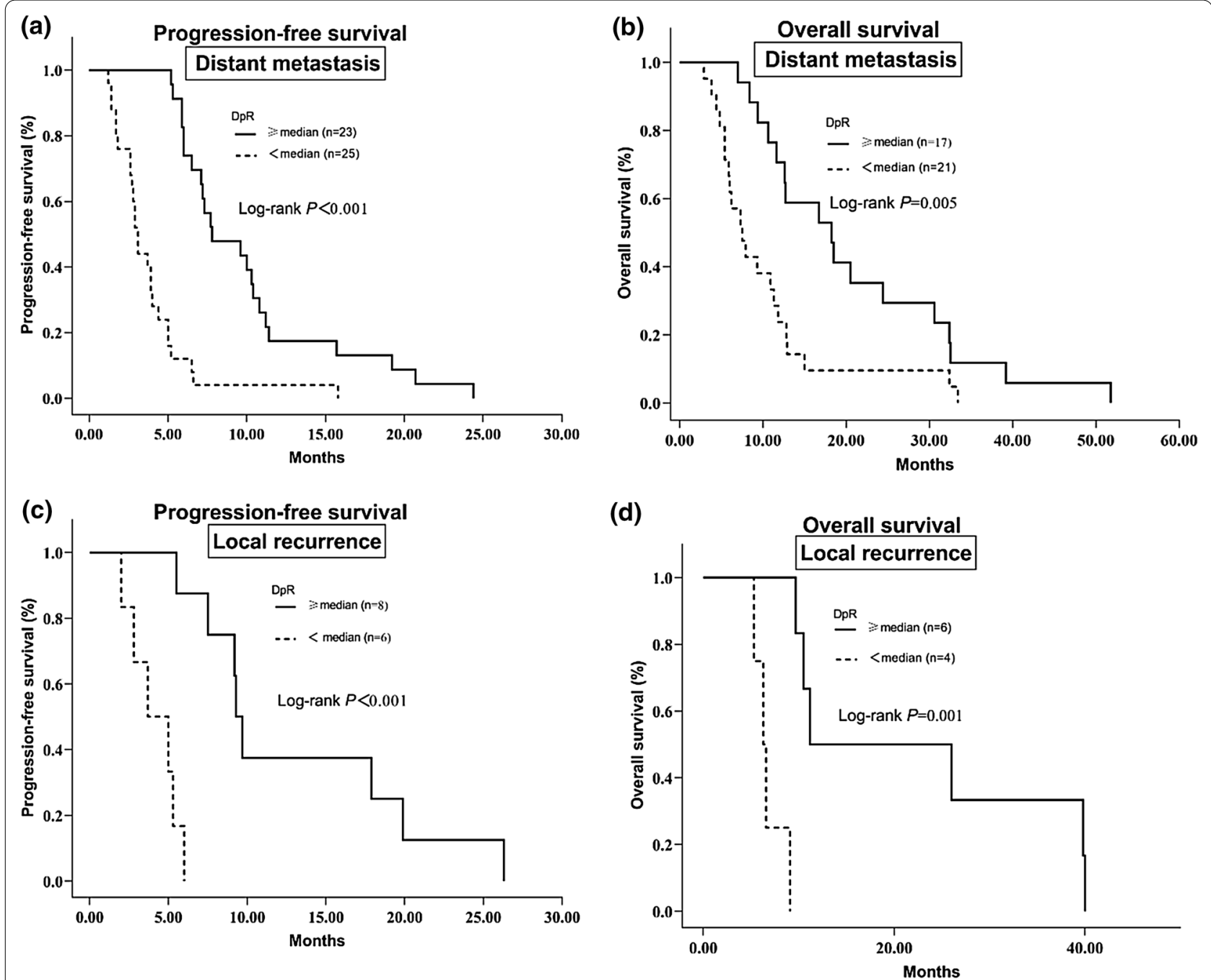

Fig. 5 a Kaplan-Meier survival curves for PFS according to DpR median value among patients with distant metastasis b Kaplan-Meier curves for OS according to DpR median value among patients with distant metastasis $\mathbf{c}$ Kaplan-Meier curves for PFS according to DpR median value among patients with local recurrence $\mathbf{d}$ Kaplan-Meier curves for OS according to DpR median value among patients with local recurrence

Table 4 Multivariate analyses

\begin{tabular}{|c|c|c|c|c|}
\hline \multirow[t]{2}{*}{ Variables } & \multicolumn{2}{|l|}{ PFS } & \multicolumn{2}{|l|}{ OS } \\
\hline & $\mathrm{HR}(95 \% \mathrm{Cl})$ & $P$-value & HR $(95 \% \mathrm{Cl})$ & $P$-value \\
\hline ECOG performance status (2) & $4.682(1.065-20.578)$ & 0.041 & - & - \\
\hline Number of metastatic sites ( $\geq 3$ ) & - & - & $6.129(1.840-20.418)$ & 0.003 \\
\hline ETS $(<20 \%)$ & - & - & $4.490(1.842-10.946)$ & 0.001 \\
\hline DpR (as a continuous variable) & $1.027(1.014-1.040)$ & $<0.001$ & $1.037(1.024-1.051)$ & $<0.001$ \\
\hline
\end{tabular}

Bold values indicate statistical significance at $P<0.05$

$D p R$ depth of response, ECOG Eastern Cooperative Oncology Group, ETS early tumour shrinkage

thus deserving further investigations. Furthermore, it is important that further studies on PC include non-ETS as a heterogeneous group.
The stratification analysis of patients with distant metastasis or local recurrence revealed that the prognostic potential of ETS was retained in patients with distant 
metastasis, while patients with local recurrence lacked any substantial link with survival (PFS and OS). This suggested that the cut-off values (20\%) set for the optimal distinction of patients with a more favorable prognosis among patients with local recurrence was not reasonable. Hubert et al. introduced the idea that further experiments may improve the prognostic potential of ETS by distinguishing between lymph nodes and organ metastases [10]. This study is the first report to investigate this parameter. However, since there were only a few patients with local recurrence (including regional lymph node metastases), additional research is necessary to verify these findings.

This study had several limitations. This is a retrospective single-center study that enrolled a limited number of patients. However, the results were consistent with previous reports that enrolled large populations. Additionally, the investigators performed the evaluations rather than by centralized radiological review, which might have introduced bias. Therefore, further large-scale prospective studies with international validation, ideally with centralized radiological assessment, is required.

\section{Conclusions}

This study indicated that an earlier and deeper tumor shrinkage could anticipate the survival of advanced PC patients. These findings need further validation before using ETS and DpR in routine procedures of patient management.

\begin{abstract}
Abbreviations
ETS: Early tumor shrinkage; DpR: Depth of response; PC: Pancreatic cancer; RMPC: Recurrent and metastatic pancreatic cancer; SLD: Sum-of-the-longestdiameters; PFS: Progression-free survival; OS: Overall survival; PPS: Postprogression survival; FOLFIRINOX: 5-Fluorouracil, irinotecan, and oxaliplatin; FOLFOXIRI: 5-Fluorouracil, irinotecan, and oxaliplatin; ECOG: Eastern cooperative oncology group; PS: Performance status; RECIST: Response evaluation criteria in solid tumors; CT: Computed tomography; HRs: Hazard ratios; Cls: Confidence intervals; GS: Gemcitabine plus S-1; GEMOX: Gemcitabine and oxaliplatin; DCR: Disease control rate; ORR: Objective response rate.
\end{abstract}

\section{Supplementary Information}

The online version contains supplementary material available at https://doi. org/10.1186/s12876-021-01870-x.

Additional file 1: Table S1: First-line treatment regimes.

\section{Acknowledgements}

We thank all of the participants and staff members involved in this study.

\section{Authors' contributions}

$\mathrm{XY}$ analyzed and interpreted the patient data regarding the ETS/DpR and the survival in RMPC and was a major contributor in writing the manuscript. $X Y$ and XX performed data collection. YW commented on previous versions of the manuscript. MQ designed and supervised this research. All authors read and approved the final manuscript.
Funding

This work did not receive any funding.

\section{Availability of data and materials}

The datasets generated and/or analyzed in the present study are available from the corresponding author on reasonable request.

\section{Declarations}

\section{Ethics approval and consent to participate}

The study was conducted in compliance with the International Conference on Harmonization guidelines for Good Clinical Practice (E6) and the 2013 Declaration of Helsinki. The study was approved by the institutional review board of Sichuan University and all patients provided written informed consent before inclusion in the study.

\section{Consent for publication}

Not applicable.

\section{Competing interests}

The authors declare that they have no competing interests.

\section{Author details}

${ }^{1}$ Division of Thoracic Oncology, Cancer Center, West China Hospital, Sichuan University, Chengdu, China. ${ }^{2}$ Division of Abdominal Oncology, Cancer Center, West China Hospital, Sichuan University, No. 37 Guo Xue Xiang, Chengdu 610041, China.

Received: 13 April 2021 Accepted: 21 June 2021

Published online: 15 July 2021

\section{References}

1. Zeng S, Pöttler M, Lan B, Grützmann R, Pilarsky C, Yang H. Chemoresistance in pancreatic cancer. Int J Mol Sci. 2019. https://doi.org/10.3390/ ijms20184504.

2. Rawla P, Sunkara T, Gaduputi V. Epidemiology of pancreatic cancer: global trends, etiology and risk factors. World J Oncol. 2019;10(1):10-27. https:// doi.org/10.14740/wjon1166.

3. Winter JM, Cameron JL, Campbell KA, Arnold MA, Chang DC, Coleman J, et al. 1423 pancreaticoduodenectomies for pancreatic cancer: a singleinstitution experience. J Gastrointest Surg Off J Soc Surg Aliment Tract. 2006;10(9):1199-210. https://doi.org/10.1016/j.gassur.2006.08.018.

4. De La Cruz MSD, Young AP, Ruffin MT. Diagnosis and management of pancreatic cancer. Am Fam Physician. 2014:89(8):626-32.

5. ASCO Cancer.Net. Pancreatic Cancer - Statistics. [cited 2020 June 30]. https://www.cancer.net/cancer-types/pancreatic-cancer/statistics.

6. Conroy T, Desseigne F, Ychou M, Bouché O, Guimbaud R, Bécouarn Y, et al. FOLFIRINOX versus gemcitabine for metastatic pancreatic cancer. N Engl J Med. 2011;364(19):1817-25. https://doi.org/10.1056/NEJMoa1011923.

7. Von Hoff DD, Ervin T, Arena FP, Chiorean EG, Infante J, Moore M, et al. Increased survival in pancreatic cancer with nab-paclitaxel plus gemcitabine. N Engl J Med. 2013;369(18):1691-703. https://doi.org/10.1056/ NEJMoa1304369.

8. Giessen C, Laubender RP, Fischer von Weikersthal L, Schalhorn A, Modest DP, Stintzing S, et al. Early tumor shrinkage in metastatic colorectal cancer: retrospective analysis from an irinotecan-based randomized first-line trial. Cancer Sci. 2013;104(6):718-24. https://doi.org/10.1111/cas.12148.

9. Mansmann U, Sartorius U, Laubender R, Giessen C, Esser R, Heinemann V. Quantitative analysis of the impact of deepness of response on post-progression survival time following first-line treatment in patients. Ann Oncol. 2013;24(suppl 4):114-5. https://doi.org/10.1093/annonc/ mdt201.9.

10. Piessevaux H, Buyse M, Schlichting M, Van Cutsem E, Bokemeyer C, Heeger $S$, et al. Use of early tumor shrinkage to predict long-term outcome in metastatic colorectal cancer treated with cetuximab. J Clin Oncol Off J Am Soc Clin Oncol. 2013;31(30):3764-75. https://doi.org/10. 1200/JCO.2012.42.8532. 
11. Heinemann V, Modest D, Fischer von Weikersthal L, Decker T, Kiani A, Vehling-Kaiser $U$, et al. Independent radiological evaluation of objective response early tumor shrinkage, and depth of response in FIRE-3 (AIO KRK-0306). Ann Oncol. 2014;25:117. https://doi.org/10.1093/annonc/ mdu193.30.

12. Cremolini C, Loupakis F, Antoniotti C, Lonardi S, Masi G, Salvatore L, et al. Early tumor shrinkage and depth of response predict long-term outcome in metastatic colorectal cancer patients treated with first-line chemotherapy plus bevacizumab: results from phase III TRIBE trial by the Gruppo Oncologico del Nord Ovest. Ann Oncol Off J Eur Soc Med Oncol. 2015;26(6):1188-94. https://doi.org/10.1093/annonc/mdv112.

13. Rivera F, Karthaus M, Hecht JR, Sevilla I, Forget F, Fasola G, et al. Final analysis of the randomised PEAK trial: overall survival and tumour responses during first-line treatment with mFOLFOX6 plus either panitumumab or bevacizumab in patients with metastatic colorectal carcinoma. Int J Colorectal Dis. 2017;32(8):1179-90. https://doi.org/10. 1007/s00384-017-2800-1.

14. Kaga Y, Sunakawa Y, Kubota Y, Tagawa T, Yamamoto T, Ikusue T, et al. Early tumor shrinkage as a predictor of favorable outcomes in patients with advanced pancreatic cancer treated with FOLFIRINOX. Oncotarget. 2016;7(41):67314-20. https://doi.org/10.18632/oncotarget.12007.

15. Vivaldi C, Fornaro L, Cappelli C, Pecora I, Catanese S, Salani F, et al. Early tumor shrinkage and depth of response evaluation in metastatic pancreatic cancer treated with first line chemotherapy: an observational retrospective cohort study. Cancers. 2019. https://doi.org/10.3390/cance rs11070939.

16. Eisenhauer EA, Therasse P, Bogaerts J, Schwartz LH, Sargent D, Ford R, et al. New response evaluation criteria in solid tumours: revised RECIST guideline (version 11). Eur J Cancer Oxf Engl. 2009;45(2):228-47. https:// doi.org/10.1016/j.ejca.2008.10.026.

17. Primavesi F, Fadinger N, Biggel S, Braunwarth E, Gasser E, Sprung S, et al. Early response evaluation during preoperative chemotherapy for colorectal liver metastases: combined size and morphology-based criteria predict pathological response and survival after resection. J Surg Oncol. 2019. https://doi.org/10.1002/jso.25796.

18. Heinemann V, Stintzing S, Modest DP, Giessen-Jung C, Michl M, Mansmann UR. Early tumour shrinkage (ETS) and depth of response (DpR) in the treatment of patients with metastatic colorectal cancer (mCRC). Eur J Cancer Oxf Engl. 2015;51(14):1927-36. https://doi.org/10.1016/j.ejca.2015. 06.116.

19. Piessevaux $H$, Buyse $M$, De Roock W, Prenen $H$, Schlichting M, Van

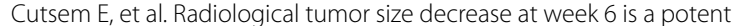
predictor of outcome in chemorefractory metastatic colorectal cancer treated with cetuximab (BOND trial). Ann Oncol Off J Eur Soc Med Oncol. 2009;20(8):1375-82. https://doi.org/10.1093/annonc/mdp011.

20. Bazarbashi S, Aljubran AH, Al Zahrani A, Edesa W, Nabil-Ahmed M, Shoukri M. Early tumor shrinkage versus early response as a predictor for overall survival (OS) in patients with metastatic colorectal cancer (mCRC) treated with triplet chemotherapy regimens (TCR). J Clin Oncol. 2014;32(15_ suppl):e14580-e14580. https://doi.org/10.1200/jco.2014.32.15_suppl. e14580.

21. Kim TW, Elme A, Park JO, Udrea AA, Kim SY, Ahn JB, et al. Final analysis of outcomes and RAS/BRAF status in a randomized phase 3 study of panitumumab and best supportive care in chemorefractory wild type KRAS metastatic colorectal cancer. Clin Colorectal Cancer. 2018;17(3):206-14 https://doi.org/10.1016/j.clcc.2018.03.008.

22. Douillard JY, Siena S, Peeters M, Koukakis R, Terwey JH, Tabernero J. Impact of early tumour shrinkage and resection on outcomes in patients with wild-type RAS metastatic colorectal cancer. Eur J Cancer. 2015;51(10):1231-42. https://doi.org/10.1016/j.ejca.2015.03.026.

\section{Publisher's Note}

Springer Nature remains neutral with regard to jurisdictional claims in published maps and institutional affiliations.
Ready to submit your research? Choose BMC and benefit from:

- fast, convenient online submission

- thorough peer review by experienced researchers in your field

- rapid publication on acceptance

- support for research data, including large and complex data types

- gold Open Access which fosters wider collaboration and increased citations

- maximum visibility for your research: over 100M website views per year

At BMC, research is always in progress.

Learn more biomedcentral.com/submissions 